\title{
LOCALLY CONFORMAL KÄHLER MANIFOLDS AND CONFORMAL SCALAR CURVATURE
}

\author{
JAEMAN KIM
}

\begin{abstract}
We show that on a compact locally conformal Kähler manifold $M^{2 n}$ ( $\left.\operatorname{dim} M^{2 n}=2 n \geq 4\right), M^{2 n}$ is Kähler if and only if its conformal scalar curvature $k$ is not smaller than the scalar curvature $s$ of $M^{2 n}$ everywhere. As a consequence, if a compact locally conformal Kähler manifold $M^{2 n}$ is both conformally flat and scalar flat, then $M^{2 n}$ is Kähler. In contrast with the compact case, we show that there exists a locally conformal Kähler manifold with $k$ equal to $s$, which is not Kähler.
\end{abstract}

\section{Introduction}

A locally conformal Kähler (lck) manifold $M^{2 n}=\left(M^{2 n}, J, g\right)\left(\operatorname{dim} M^{2 n}=\right.$ $2 n \geq 4$ ) is a Hermitian manifold (i.e., the metric $g$ of $M^{2 n}$ is compatible with complex structure $J$ ) whose metric $g$ is locally conformal to a Kähler metric, which is equivalent to the existence of an 1-form $\theta$ (called Lee form) of $M^{2 n}$ such that $d \Omega=\theta \wedge \Omega$ and $d \theta=0$ [10], [11], where $\Omega$ is the Kähler form of $M^{2 n}$. From the viewpoint of conformal geometry, we can define a conformally well-behaved function (namely, conformal scalar curvature) $k$ on $M^{2 n}$ associated with Weyl curvature tensor, which appeared in the literature of Hermitian geometry [1], [4], [5]. It is well known that the standard Hopf surface $M^{4}$ is a compact lck manifold with positive scalar curvature $s$ and vanishing conformal scalar curvature $k$, which is not Kähler. In this note, we investigate a condition of the conformal scalar curvature $k$ for a compact lck manifold $M^{2 n}$ to be Kähler and show that the sign of difference between $k$ and $s$ is a crucial condition for a compact lck manifold $M^{2 n}$ to be Kähler. More precisely, we prove the following:

Received July 10, 2009

2000 Mathematics Subject Classification. 53A30, 53B35, 53C25, 53C55, 53C56.

Key words and phrases. compact locally conformal Kähler manifold, conformal scalar curvature, Kähler, conformally flat and scalar flat, a locally conformal Kähler manifold with $k$ equal to $s$.

This study is supported by Kangwon National University. 
Theorem 1.1. Let $M^{2 n}=\left(M^{2 n}, J, g\right)$ be a compact lck manifold. Then $M^{2 n}$ is Kähler if and only if its conformal scalar curvature $k$ is not smaller than the scalar curvature $s$ of $M^{2 n}$ everywhere.

As a consequence, we have:

Corollary 1.2. If a compact lck manifold $M^{2 n}$ is conformally flat, and its scalar curvature vanishes, then $M^{2 n}$ is Kähler.

Contrary to the compact case, we obtain the following:

Theorem 1.3. Let $R_{+}^{2 n}=\left\{\left(x_{1}, x_{2}, \ldots, x_{2 n}\right) \mid x_{2 n}>0\right\}$ and $J$ be the natural complex structure defined by $J\left(\frac{\partial}{\partial x_{2 i-1}}\right)=\frac{\partial}{\partial x_{2 i}}, J\left(\frac{\partial}{\partial x_{2 i}}\right)=-\frac{\partial}{\partial x_{2 i-1}}$. And a metric $g=\left(g_{i j}\right)$ on $R_{+}^{2 n}$ is given by $g_{i j}=x_{2 n}^{\frac{4}{2 n-2}} \delta_{i j}$. Then $M=\left(R_{+}^{2 n}, J, g\right)$ is a lck manifold with $k=s$, which is not Kähler.

\section{Preliminaries}

We shall denote by $M^{2 n}=\left(M^{2 n}, J, g\right)\left(\operatorname{dim} M^{2 n}=2 n \geq 4\right)$ a lck manifold; by $g$ its metric; by $\left\{U_{\alpha}\right\}$ an open covering of $M^{2 n}$ endowed with smooth functions $f_{\alpha}$ on $U_{\alpha}$ such that $\widetilde{g_{\alpha}}=e^{-f_{\alpha}} g$ are Kähler metrics; by $J$ the complex structure; by $\nabla$ the Levi-Civita connection of $g$; by $\theta$ the Lee form of $M^{2 n}$, which satisfies $d \Omega=\theta \wedge \Omega$ and $d \theta=0$, where $\Omega$ is the Kähler form of $M^{2 n}$, i.e., $g(X, J Y)=\Omega(X, Y)$. Note that $M^{2 n}$ is Kähler if and only if $\theta=0$. The Riemannian curvature tensor $R$, the Ricci tensor Ric and the scalar curvature $s$ are given by

$$
\begin{gathered}
R(X, Y) Z=\left[\nabla_{X}, \nabla_{Y}\right] Z-\nabla_{[X, Y]} Z \\
R i c(X, Y)=\text { Trace }\{Z \rightarrow R(Z, X) Y\}, s=\text { Trace }_{g} \text { Ric. }
\end{gathered}
$$

Furthermore, the $*$-Ricci tensor and $*$-scalar curvature of $(J, g)$ are given by

$$
\operatorname{Ric}^{*}(X, Y)=\operatorname{Trace}\{Z \rightarrow-J R(Z, X) J Y\}, s^{*}=\text { Trace }_{g} \operatorname{Ric}^{*} .
$$

The Riemannian metric $g$ induces a metric on the bundle $\Lambda^{2}$ of 2-vectors on $M^{2 n}$ by

$$
\left\langle X_{k} \wedge X_{l}, Y_{p} \wedge Y_{q}\right\rangle=\operatorname{det}\left(g\left(X_{i}, Y_{j}\right)\right) .
$$

Similarly, one can define a metric on the bundle $\bigwedge^{2}$ of 2 -forms on $M^{2 n}$ by

$$
\langle A \wedge B, C \wedge D\rangle=\left\langle A^{\sharp} \wedge B^{\sharp}, C^{\sharp} \wedge D^{\sharp}\right\rangle \text {. }
$$

Here the symbol $\sharp$ is the natural isomorphism from 1 -forms to vector fields. We also regard the curvature tensor as a $(0,4)$-tensor or an endomorphism of the 2-form bundle as follows:

$$
R(X, Y, Z, V)=-g(R(X, Y) Z, V),\langle R(A \wedge B), C \wedge D\rangle=R\left(A^{\sharp}, B^{\sharp}, C^{\sharp}, D^{\sharp}\right) .
$$

The Riemannian curvature tensor $R$ has the following well known $S O(2 n)$ decomposition [2], [6], [7];

$$
R=\frac{s}{4 n(2 n-1)} g \star g+\frac{1}{2 n-2} R i c_{o} \star g+W,
$$


where $\operatorname{Ric}_{o}$ is the traceless Ricci tensor and $W$ is the Weyl curvature tensor. Here the symbol $\star$ is the Nomizu-Kulkarni product of symmetric $(0,2)$-tensors generating a curvature type tensor. Note that $R i c_{o}=0$ if and only if $M^{2 n}$ is Einstein [2], [7], [8]; $W=0$ if and only if $M^{2 n}$ is conformally flat [2], [6]. Now we define the conformal scalar curvature $k$ of $M^{2 n}$ by

$$
k=\frac{2 n-1}{n-1}\langle W(\Omega), \Omega\rangle .
$$

In particular, if $2 n=4$, then $k=3\left\langle W^{+}(\Omega), \Omega\right\rangle$, which appeared in the literature of Hermitian geometry [1], [4]. Therefore, if $M^{2 n}$ is conformally flat, then the conformal scalar curvature $k$ of $M^{2 n}$ is zero. Note that $k$ has conformal weight -2 , that is, if we replace $g$ by $f^{2} g$ for some non-vanishing function $f$, then $k$ is replaced by $f^{-2} k$.

\section{Proof of Theorem 1.1}

Let $M^{2 n}=\left(M^{2 n}, J, g\right)$ be a compact lck manifold. From the relation $(2.1)$ and $\left\langle\left(R i c_{o} \star g\right)(\Omega), \Omega\right\rangle=0$, we obtain

$$
\langle R(\Omega), \Omega\rangle=\frac{s}{4 n(2 n-1)} 2\langle\Omega, \Omega\rangle+\langle W(\Omega), \Omega\rangle .
$$

Therefore, from $\langle\Omega, \Omega\rangle=n$ and the definitions of $s^{*}$ and $k$, the above identity yields

which implies

$$
\frac{s^{*}}{2}=\frac{s}{2(2 n-1)}+\frac{n-1}{2 n-1} k,
$$

$$
s-k=\frac{2 n-1}{2 n-2}\left(s-s^{*}\right) .
$$

Now suppose that $M^{2 n}$ is Kähler and hence $s$ and $s^{*}$ coincide; this is a consequence of the Kähler identity [2] $R(X, Y)(J Z)=J(R(X, Y) Z)$, which itself follows from the fact that $\nabla J=0$. Therefore, the equation (3.2) implies $k=s$. Conversely, let a compact lck manifold $M^{2 n}$ satisfy $k \geq s$. And assume that $\widetilde{g}=e^{-f} g$ is the lck metric of $g$. Then one gets the well-known formula [3], [9]

$$
\begin{aligned}
e^{f} \widetilde{g}(\widetilde{R}(X, Y) Z, W)= & g(R(X, Y) Z, W)-\frac{1}{2}\{L(X, Z) g(Y, W)-L(Y, Z) g(X, W) \\
& +L(Y, W) g(X, Z)-L(X, W) g(Y, Z)\} \\
& -\frac{\|\theta\|^{2}}{4}\{g(Y, Z) g(X, W)-g(X, Z) g(Y, W)\},
\end{aligned}
$$

which yields

$$
\begin{aligned}
& g(R(X, Y) Z, W)-g(R(X, Y) J Z, J W) \\
= & \frac{1}{2}\{L(X, Z) g(Y, W)-L(Y, Z) g(X, W)+L(Y, W) g(X, Z)-L(X, W) g(Y, Z)\} \\
& +\frac{\|\theta\|^{2}}{4}\{g(Y, Z) g(X, W)-g(X, Z) g(Y, W)\}
\end{aligned}
$$




$$
\begin{aligned}
& -\frac{1}{2}\{L(X, J Z) g(Y, J W)-L(Y, J Z) g(X, J W) \\
& +L(Y, J W) g(X, J Z)-L(X, J W) g(Y, J Z)\} \\
& -\frac{\|\theta\|^{2}}{4}\{g(Y, J Z) g(X, J W)-g(X, J Z) g(Y, J W)\},
\end{aligned}
$$

where $L(X, Y)=\left(\nabla_{X} \theta\right) Y+\frac{1}{2} \theta(X) \theta(Y)$ and so $L(X, Y)=L(Y, X)$ since $\theta$ is closed. Now if we take $X=\frac{\partial}{\partial x_{i}}, Y=\frac{\partial}{\partial x_{j}}, Z=\frac{\partial}{\partial x_{k}}, W=\frac{\partial}{\partial x_{l}}$, where $x_{i}$ $(i=1, \ldots, 2 n)$ are real coordinates on $M^{2 n}$, and contract with $g^{i l} g^{j k}$, and then use the identity (3.2), we get the following [3], [5], [9]:

$$
s-k=\frac{2 n-1}{2 n-2}\left(2(n-1) \delta \theta+(n-1)^{2}\|\theta\|^{2}\right),
$$

where $\delta \theta=-\operatorname{div} \theta$.

By integrating (3.3) over $M^{2 n}$, we conclude that the condition of $k \geq s$ yields $\theta=0$. Summing up the above arguments, we conclude that on a compact lck manifold, the Kähler condition is equivalent to that of $k \geq s$. This completes the proof of Theorem 1.1 and hence it is obvious that Corollary 1.2 holds because of $k=s$.

\section{A lck manifold with $k$ equal to $s$ which is not Kähler}

Let $R_{+}^{2 n}=\left\{\left(x_{1}, x_{2}, \ldots, x_{2 n}\right) \mid x_{2 n}>0\right\}$ and $J$ be the natural complex structure defined by $J\left(\frac{\partial}{\partial x_{2 i-1}}\right)=\frac{\partial}{\partial x_{2 i}}, J\left(\frac{\partial}{\partial x_{2 i}}\right)=-\frac{\partial}{\partial x_{2 i-1}}$. We define a Riemannian metric $g=\left(g_{i j}\right)$ on $R_{+}^{2 n}$ by $g_{i j}=x_{2 n}^{\frac{4}{2 n-2}} \delta_{i j}$. It is obvious that the metric $g$ is compatible with complex structure $J$. With respect to $\left\{\frac{\partial}{\partial x_{1}}, \frac{\partial}{\partial x_{2}}, \ldots, \frac{\partial}{\partial x_{2 n}}\right\}$, we set $\nabla_{\frac{\partial}{\partial x_{i}}} \frac{\partial}{\partial x_{j}}=\sum_{k=1, \ldots, 2 n} \Gamma_{i j}^{k} \frac{\partial}{\partial x_{k}}$ for $1 \leq i, j \leq 2 n$. Then the Christoffel symbol $\Gamma_{i j}^{k}$ of metric $g$ is obtained as follows:

$$
\begin{aligned}
\Gamma_{1(2 n)}^{1} & =\Gamma_{(2 n) 1}^{1}=\Gamma_{2(2 n)}^{2}=\Gamma_{(2 n) 2}^{2}=\cdots \\
& =\Gamma_{(2 n-1) 2 n}^{2 n-1}=\Gamma_{2 n(2 n-1)}^{2 n-1}=\frac{2}{2 n-2}\left(\frac{1}{x_{2 n}}\right), \\
\Gamma_{2 n(2 n)}^{2 n} & =\frac{2}{2 n-2}\left(\frac{1}{x_{2 n}}\right), \Gamma_{11}^{2 n}=\Gamma_{22}^{2 n}=\cdots \\
& =\Gamma_{(2 n-1)(2 n-1)}^{2 n}=-\frac{2}{2 n-2}\left(\frac{1}{x_{2 n}}\right)
\end{aligned}
$$

and are otherwise zero. And so we have

$$
\begin{aligned}
R_{121}^{2} & =R_{131}^{3}=\cdots=R_{1(2 n-1) 1}^{2 n-1}=R_{212}^{1}=R_{232}^{3}=\cdots=R_{2(2 n-1) 2}^{2 n-1} \\
& =\cdots=R_{(2 n-2) 1(2 n-2)}^{1}=R_{(2 n-2) 2(2 n-2)}^{2}=\cdots \\
& =R_{(2 n-2)(2 n-1)(2 n-2)}^{2 n-1}=R_{(2 n-1) 1(2 n-1)}^{1}=R_{(2 n-1) 2(2 n-1)}^{2} \\
& =\cdots=R_{(2 n-1)(2 n-2)(2 n-1)}^{2 n-2}=-\frac{4}{(2 n-2)^{2}}\left(\frac{1}{x_{2 n}^{2}}\right),
\end{aligned}
$$




$$
\begin{aligned}
R_{(2 n) 1(2 n)}^{1} & =R_{1(2 n) 1}^{2 n}=R_{(2 n) 2(2 n)}^{2}=R_{2(2 n) 2}^{2 n}=R_{(2 n) 3(2 n)}^{3} \\
& =R_{3(2 n) 3}^{2 n}=\cdots=R_{(2 n-1)(2 n)(2 n-1)}^{2 n}=\frac{2}{2 n-2}\left(\frac{1}{x_{2 n}^{2}}\right)
\end{aligned}
$$

and are otherwise zero. Here $R_{j l k}^{p}=g^{p i} R_{i j l k}$ and $R_{i j l k}=g\left(R\left(\frac{\partial}{\partial x_{i}}, \frac{\partial}{\partial x_{j}}, \frac{\partial}{\partial x_{k}}\right), \frac{\partial}{\partial x_{l}}\right)$. Hence we get

$$
s=s^{*}=0,
$$

which yields from the equation $(3.2)$

$$
s=k \text {. }
$$

On the other hand, we have

$$
d \Omega=\frac{4}{2 n-2}\left(\frac{1}{x_{2 n}}\right) d x_{2 n} \wedge \Omega,
$$

which implies that the Lee form $\theta=\frac{4}{2 n-2}\left(\frac{1}{x_{2 n}}\right) d x_{2 n}$ of $M^{2 n}$ is closed. Summing up the above arguments, we obtain that $R_{+}^{2 n}$ allows a lck structure $(J, g)$ with $k=s$, which is not Kähler. This completes the proof of Theorem 1.3.

\section{References}

[1] V. Apostolov and P. Gauduchon, The Riemannian Goldberg-Sachs theorem, Internat. J. Math. 8 (1997), no. 4, 421-439.

[2] A. L. Besse, Einstein Manifolds, Springer-Verlag, Berlin, 1987.

[3] S. Dragomir and L. Ornea, Locally Conformal Kähler Geometry, Progress in Mathematics, 155. Birkhäuser Boston, Inc., Boston, MA, 1998.

[4] P. Gauduchon and S. Ivanov, Einstein-Hermitian surfaces and Hermitian Einstein-Weyl structures in dimension 4, Math. Z. 226 (1997), no. 2, 317-326.

[5] P. Gauduchon, La 1-forme de torsion d'une variété hermitienne compacte, Math. Ann. 267 (1984), no. 4, 495-518.

[6] Z. Hu, H. Li, and U. Simon, Schouten curvature functions on locally conformally flat Riemannian manifolds, J. Geom. 88 (2008), no. 1-2, 75-100.

[7] J. Kim, Rigidity theorems for Einstein-Thorpe metrics, Geom. Dedicata 80 (2000), no. $1-3,281-287$.

[8] _ On Einstein Hermitian manifolds, Monatsh. Math. 152 (2007), no. 3, 251-254.

[9] I. Vaisman, Some curvature properties of complex surfaces, Ann. Mat. Pura Appl. (4) 132 (1982), 1-18.

[10] Some curvature properties of locally conformal Kähler manifolds, Trans. Amer. Math. Soc. 259 (1980), no. 2, 439-447.

[11] A theorem on compact locally conformal Kähler manifolds, Proc. Amer. Math. Soc. 75 (1979), no. 2, 279-283.

Department of Mathematics Education

KANGWON NATIONAL UNIVERSITY

Chunchon 200-701, Korea

E-mail address: jaeman64@kangwon.ac.kr 